\title{
Variable Ordering for the Application of BDDs to the Maximum Independent Set Problem
}

\author{
David Bergman, Andre A. Cire, Willem-Jan van Hoeve, J. N. Hooker \\ Tepper School of Business, Carnegie Mellon University \\ 5000 Forbes Ave., Pittsburgh, PA 15213, U.S.A. \\ \{dbergman, acire, vanhoeve\}@andrew.cmu.edu, john@hooker.tepper.cmu.edu *
}

\begin{abstract}
The ordering of variables can have a significant effect on the size of the reduced binary decision diagram (BDD) that represents the set of solutions to a combinatorial optimization problem. It also influences the quality of the objective function bound provided by a limited-width relaxation of the BDD. We investigate these effects for the maximum independent set problem. By identifying variable orderings for the BDD, we show that the width of an exact BDD can be given a theoretical upper bound for certain classes of graphs. In addition, we draw an interesting connection between the Fibonacci numbers and the width of exact BDDs for general graphs. We propose variable ordering heuristics inspired by these results, as well as a k-layer look-ahead heuristic applicable to any problem domain. We find experimentally that orderings that result in smaller exact BDDs have a strong tendency to produce tighter bounds in relaxation BDDs.
\end{abstract}

\section{Introduction}

In recent years, Binary Decision Diagrams (BDDs) $[1,17,7]$ have been regarded as a powerful tool for a variety of purposes in Operations Research. Their application in this domain is primarily as a graphical data structure that aims at a compact representation of the set of feasible solutions to a Constraint Satisfaction Problem (CSP). Examples of applications include the generation of cuts in a Branch-and-cut framework [3], post-optimality analysis for Integer Programming $[12,13]$, and $0 / 1$ vertex and facet enumeration [4].

This perspective of BDDs is nonetheless associated with inherent difficulties. First, constructing the exact BDD for a CSP instance is in general an NP-hard problem, since this procedure is as hard as deciding the feasibility of the instance. Furthermore, even for problems where feasibility can be determined efficiently, the BDD may grow exponentially large, and thus it is not computationally practical to derive exact representations for most relevant problems.

In light of these difficulties, the work in [2] proposes the use of limited-size BDDs as an approximate representation for CSPs. Namely, limited-size BDDs are

\footnotetext{
* This work was supported by NSF under grant CMMI-1130012 and AFOSR under grant FA-95501110180.
} 
constructed in a way that they contain the feasible space of a particular CSP, but may potentially include infeasible solutions due to the imposition of a polynomial bound on their size according to an input parameter. Such approximations have led to research along several directions, such as replacing the traditional domain store relaxation used in Constraint Programming systems [2, 14, 15].

In this context, the work in [5] introduced a systematic approach to generate approximate BDDs for Combinatorial Optimization problems. The authors focused on using BDDs to represent a relaxation of the set of feasible solutions to the Set Covering Problem (SCP). These structures were introduced for the purpose of proving bounds on the objective function for the SCP. It also proposes methods for tightening the bound provided by the relaxed BDDs, analogous to the use of cutting planes in Integer Programming (IP). It was shown by the authors that for structured instances of the SCP, the relaxations based on BDDs can provide substantially tighter bounds than the classical linear relaxation.

We further extend this line of research in the present paper, investigating one of the crucial aspects of BDDs applied to Operations Research problems: How does the ordering of the variables within a BDD affect the quality of the relaxation it provides. In particular, we are interested in identifying the relation between the size of an exact BDD for a CSP, which is directly correlated to the variable ordering applied, and the bounds obtained through its corresponding approximate BDD when an objective function is considered.

The development of good variable orderings requires identifying the underlying cause of the combinatorial explosion of the size of exact BDDs. For this purpose, this work focuses on the Maximum Independent Set Problem (MISP), exploring the following main topics. We first introduce a technique to efficiently construct the BDD representing the family of independent sets of a graph. Next, we provide a thorough study of orderings that yield polynomially-bounded BDD sizes for particular classes of graphs. Through this analysis we uncover an interesting connection between the size of exact BDDs for arbitrary graphs and the Fibonacci numbers, yet another curious property of independent sets $[8,11$, $9,18]$. Interestingly, we illustrate how the underlying principles in the proof of these bounds on the width can be used to develop good ordering heuristics. Finally, we experimentally show in this paper that variable orderings that yield small-sized exact BDDs are critical for their application as a bounding technique to optimization problems, resulting in substantially better bounds for the MISP when compared to other orderings.

The contributions of this work potentially go beyond the scope of independent set problems. Namely, we presented the first systematic analysis and empirical evidence of how variable orderings can positively affect approximate BDDs in Combinatorial Optimization. This analysis may be extended to various other problem domains. We particularly reinforce the claim that investigating orderings for particular problem classes can lead to good heuristics that are potentially applicable to other problems. In particular, we introduce the general-purpose variable ordering heuristic $k$-stage lookahead, that yielded the best results for the MISP and can be directly used for any CSP. 
This paper is organized as follows. In Section 2 we formally introduce BDDs. In Section 3 we discuss how exact BDDs for the MISP can be constructed. In Section 4 we investigate variable ordering for particular classes of instances of the MISP and prove bounds on the size of the exact BDDs for these problems. In Section 5 we discuss variable ordering heuristics for general graphs. Finally, in Section 6 we provide computational results and conclude in Section 7.

\section{Preliminaries and Notation}

CSPs A CSP $(X, D, \mathcal{C})$ is defined by a finite set of variables $X$, a set of discrete domains $D$ such that $D(x) \in D$ restricts the values $x \in X$ can take, and a set of constraints $\mathcal{C}$. A solution to a CSP corresponds to an assignment of values to the variables and it is feasible if all values are within the variable domains and are consistent with $\mathcal{C}$. A Constraint Optimization Problem (COP) is given by a CSP alongside an objective function $f$ to be maximized. For simplicity, we consider here only CSPs with binary domains, i.e., $D(x)=\{0,1\}$ for all $x \in X$.

BDDs We are interested in representing the set of solutions of a CSP by a Binary Decision Diagram (BDD). A BDD $B=(U, A, d)$ is a directed acyclic graph whose nodes $U$ are partitioned into $m$ layers, $U=\cup_{i=1}^{m} L_{i}$. The layer of a node $u$ is given by $\operatorname{var}(u)$. Layers $L_{1}$ and $L_{m}$ consist of single nodes; the root $r$ and the terminal $t$, respectively. The width $\omega_{j}$ of a layer $j$ is defined as $\omega_{j}:=\left|L_{j}\right|$, and the width of $B$ is given by $\omega(B):=\max _{j} \omega_{j}$. Let $|B|=|U|$ be the size of the BDD. All arcs $a \in A$ are directed from nodes in layer $j$ to nodes in layer $j+1$, for some $j \in\{1, \ldots, m-1\}$. The function $d: A \rightarrow\{0,1\}$ associates each arc $a$ with a label $d(a)=d_{a} \in\{0,1\} ; a$ is referred to as a one-arc if $d_{a}=1$ and as a zero-arc if $d_{a}=0$. Each node $u$ can have at most one one-arc and at most one zero-arc directed out of it. For any node $u$, there must exist a directed path from $r$ to $u$ and from $u$ to $t$. A BDD representing a set of solutions of a $\operatorname{CSP}(X, D, \mathcal{C})$, with $n=|X|$, has $m=n+1$ layers. Each layer $L_{i}$ is uniquely associated with a variable $x \in X$; we denote this variable by $x_{i}$. An arc $a$ directed from layer $L_{i}$ to $L_{i+1}$ with label $d_{a}$ identifies an assignment $x_{i}=d_{a}$. Hence, a directed path from $r$ to $t$ corresponds to a solution of the CSP. The set of solutions represented by a $\operatorname{BDD} B$ (i.e., on all directed paths from $r$ to $t$ ) is denoted by $\operatorname{Sol}(B)$.

For a given node $u \in U$, we let $\left.B^{+}\right|_{u}$ be the subgraph of $B$ induced by the subset of nodes composed of $u$, the root $r \in U$, and all nodes $v \in U$ lying on some directed path from $r$ to $u$. In addition, we preserve the arc labels as in $B$; therefore, $\left.B^{+}\right|_{u}$ is also a BDD. Analogously, let $\left.B^{-}\right|_{u}$ be the subgraph of $B$ induced by the subset of nodes composed by $u$, the terminal $t \in U$, and all nodes $v \in U$ such that there is a directed path from $u$ to $t$. Also, let $\left.B^{+}\right|_{L_{j}}$ be the digraph induced by $L_{1}, \ldots, L_{j}$ and similarly $\left.B^{+}\right|_{L_{j}}$ be the digraph induced by $L_{j}, \ldots, L_{n+1}$, with $\operatorname{Sol}\left(\left.B^{+}\right|_{L_{j}}\right)=\cup_{u \in L_{j}} \operatorname{Sol}\left(\left.B^{+}\right|_{u}\right)$ and $\operatorname{Sol}\left(\left.B^{-}\right|_{L_{j}}\right)=\cup_{u \in L_{j}} \operatorname{Sol}\left(\left.B^{-}\right|_{u}\right)$. A reduced $\operatorname{BDD}$ is one for which $\operatorname{Sol}\left(\left.B^{-}\right|_{u}\right) \neq \operatorname{Sol}\left(\left.B^{-}\right|_{u^{\prime}}\right)$ for any two nodes $u$ and $u^{\prime}$ on the same layer. It can be shown that for a particular ordering of the variables, that is, how layers are mapped into variables, there is one unique reduced BDD for any set of solutions [7]. 
MISP In this paper we study variable orderings for the Maximum Independent Set Problem (MISP). Let $G=(V, E)$ be a simple undirected graph. An independent set of $G$ is a set $I \subseteq V$ such that $(w, v) \notin E$ for any distinct $w, v \in I$. We denote $\mathcal{I}(G)$ as the family of independent sets in $G$. The MISP consists of finding a set $I \in \mathcal{I}(G)$ with the largest cardinality.

BDD representation for MISP For notation purposes, let $G[W]$ be the graph induced by a subset $W \subseteq V$ and let $\bar{W}:=V \backslash W$. A corresponding BDD for the COP above defines a bijection between the vertices $v \in V$ and the layers $L_{1}, \ldots, L_{n}$; let $v_{j}$ be the associated layer of vertex $v$, with $V_{j}=\left\{v_{1}, \ldots, v_{j}\right\}$. With every path $p=\left(a_{1}, \ldots, a_{n}\right)$ from the root $r$ to the terminal $t$ we associate a subset $I^{p} \subseteq V$ defined by $I^{p}:=\left\{v_{j}: d_{a_{j}}=1\right\}$. Likewise, for a node $u$, any path $p=\left(a_{1}, \ldots, a_{j-1}\right)$ in $\left.B^{+}\right|_{u}$ corresponds to a vertex subset in $G\left[V_{j-1}\right]$ and any path $p=\left(a_{j}, \ldots, a_{n}\right)$ in $\left.B^{-}\right|_{u}$ corresponds to a vertex subset in $G\left[\bar{V}_{j-1}\right]$. Note that each solution corresponds to at most one path in any BDD because no node has two arcs with the same label directed out of it.

A BDD $B$ is exact for a graph $G$ if $\operatorname{Sol}(B)=\mathcal{I}(G)$, and it is a relaxation for $G$ if $\operatorname{Sol}(B) \supseteq \mathcal{I}(G)$. In an exact BDD, $\mathcal{I}\left(G\left[V_{j-1}\right]\right)=\operatorname{Sol}\left(\left.B^{+}\right|_{L_{j}}\right)$ and $\mathcal{I}\left(G\left[\bar{V}_{j-1}\right]\right)=$ $\operatorname{Sol}\left(\left.B^{-}\right|_{L_{j}}\right)$. In a relaxation BDD, we similarly have $\mathcal{I}\left(G\left[V_{j-1}\right]\right) \subseteq \operatorname{Sol}\left(\left.B^{+}\right|_{L_{j}}\right)$ and $\mathcal{I}\left(G\left[\bar{V}_{j-1}\right]\right) \subseteq \operatorname{Sol}\left(\left.B^{-}\right|_{L_{j}}\right)$.

By associating a cost $d_{a}$ to each arc in a BDD $B$, the longest path from $r$ to $t$ yields a maximum cardinality independent set of $G$, if $B$ is an exact BDD. If otherwise $B$ is a relaxation $\mathrm{BDD}$, the longest path corresponds to a subset of the vertices whose cardinality is greater than or equal to the size of the maximum independent set, thereby establishes an upper-bound on the value of the optimal solution to the MISP on $G$. We note that since BDDs are layered graphs, the longest path can be computed in polynomial time in $|B|$.

Additional Notation For a graph $G=(V, E)$, two disjoint subsets $I, J \subset V$ are independent if $(w, v) \notin E$ for any $w \in I, v \in J$. The neighborhood $N(v)$ of $v \in V$ is defined as $N(v)=\{w:(w, v) \in E\}$. A partial solution with respect to $W \subseteq V$ corresponds to any subset $I \subseteq W$, which is feasible if $I \in \mathcal{I}(G[W])$. Given a partial feasible solution $I$ with respect to $W$, the set of feasible completions of $I$ with respect to $W$ is given by $C(I \mid \bar{W})=\{J \mid J \subseteq \bar{W}, I \cup J \in \mathcal{I}(G)\}$.

Example 1. Consider the MISP on the graph in Figure 1. An exact BDD representation of the feasible set is given next to the graph, where arc $(u, v)$ is solid or dashed if it is labelled as 1 or 0, respectively. Assigning arc costs as described above yields a longest path with value 3 in the BDD.

\section{Exact BDD Compilation}

A general method for creating exact BDDs for CSPs, known as top-down compilation, is presented in [5]. It consists of constructing layers $L_{1}, \ldots, L_{n}$ in that order, adding one node at a time at each layer. A node $u$ is removed if the paths from $r$ to $u$ do not correspond to feasible partial solutions (i.e., $u$ is infeasible), 

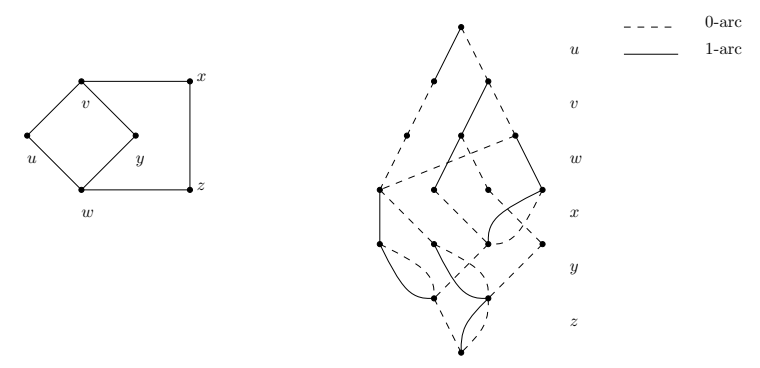

Fig. 1: Example of the exact BDD for a graph $G$.

and two nodes $u, w$ are merged if all the partial solutions on paths from $r$ to $u$ and from $r$ to $w$ have the same set of feasible completions (i.e., $u$ and $w$ are equivalent). The key of this technique is that the infeasibility and equivalence conditions are determined efficiently through the analysis of a state associated with each node, which is defined according to the problem constraints.

In order to apply the top-down exact BDD compilation algorithm for the MISP, we first establish a condition for identifying when two independent sets $I_{1}, I_{2} \in \mathcal{I}\left(G\left[V_{j-1}\right]\right)$ have the same set of feasible completions.

Theorem 1. Given a graph $G=(V, E)$, a subset $\left\{v_{1}, \ldots, v_{j-1}\right\}=V_{j-1} \subseteq V$ of the vertices of $G$, and two independent sets $I_{1}, I_{2} \subseteq \mathcal{I}\left(G\left[V_{j-1}\right]\right)$,

$$
C\left(I_{1} \mid \bar{V}_{j-1}\right)=C\left(I_{2} \mid \bar{V}_{j-1}\right) \Longleftrightarrow \bar{V}_{j-1} \backslash \cup_{v \in I_{1}} N(v)=\bar{V}_{j-1} \backslash \cup_{v \in I_{2}} N(v) .
$$

Proof. For $I \in \mathcal{I}\left(G\left[V_{j-1}\right]\right)$, we must have $C\left(I \mid \bar{V}_{j-1}\right)=\mathcal{I}\left(G\left[\bar{V}_{j-1} \backslash \cup_{v \in I_{1}} N(v)\right]\right)$, since $\bar{V}_{j-1} \backslash \cup_{v \in I_{1}} N(v)$ is exactly the set of remaining vertices in $G$ that are independent of $I$. Conversely, suppose $\bar{V}_{j-1} \backslash \cup_{v \in I_{1}} N(v) \neq \bar{V}_{j-1} \backslash \cup_{v \in I_{2}} N(v)$. Without loss of generality, suppose there exists some $w \in \bar{V}_{j-1} \backslash \cup_{v \in I_{1}} N(v)$ that is not in $\bar{V}_{j-1} \backslash \cup_{v \in I_{2}} N(v)$. Then, $w \in C\left(I_{1} \mid \bar{V}_{j-1}\right)$ but $w \notin C\left(I_{1} \mid \bar{V}_{j-1}\right)$, hence $\{v\} \cup I_{1}$ is an independent set while $\{v\} \cup I_{2}$ is not, concluding the proof.

Let $E\left(I \mid \bar{V}_{j-1}\right):=\bar{V}_{j-1} \backslash \cup_{v \in I} N(v)$ be the set of eligible vertices of $I \in$ $\mathcal{I}\left(G\left[V_{j-1}\right]\right)$; i.e., the vertices $v \in \bar{V}_{j-1}$ for which $I \cup\{v\}$ is an independent set. According to Theorem 1, we can directly use the eligible vertex set as the state for each node in order to perform the top-down compilation. Namely, since two independent sets $I_{1}, I_{2} \in \mathcal{I}\left(G\left[V_{j}\right]\right)$ with the same set of feasible completions have $E\left(I_{1} \mid \bar{V}_{j}\right)=E\left(I_{2} \mid \bar{V}_{j}\right)$, we label a BDD node $u \in L_{j}$ with a state $E(u)=E\left(I \mid \bar{V}_{j}\right)$ for any $I \in \operatorname{Sol}\left(\left.B^{+}\right|_{u}\right)$, as they must be all equal for any such $I$. Hence, all paths from $r$ to $u$ correspond to partial solutions with the same set of feasible completions. A node $u$ can only have an one-arc directed out of it if $v_{j} \in E(u)$ (infeasibility), and two nodes $u$ and $w$ are equivalent if $E(u)=E(w)$. These tests are complete, i.e., they are necessary and sufficient to determine when a node is infeasible or two nodes are equivalent. Thus, as noted in [5], the top-down compilation using the tests above yields the reduced BDD with respect to a particular variable ordering. 


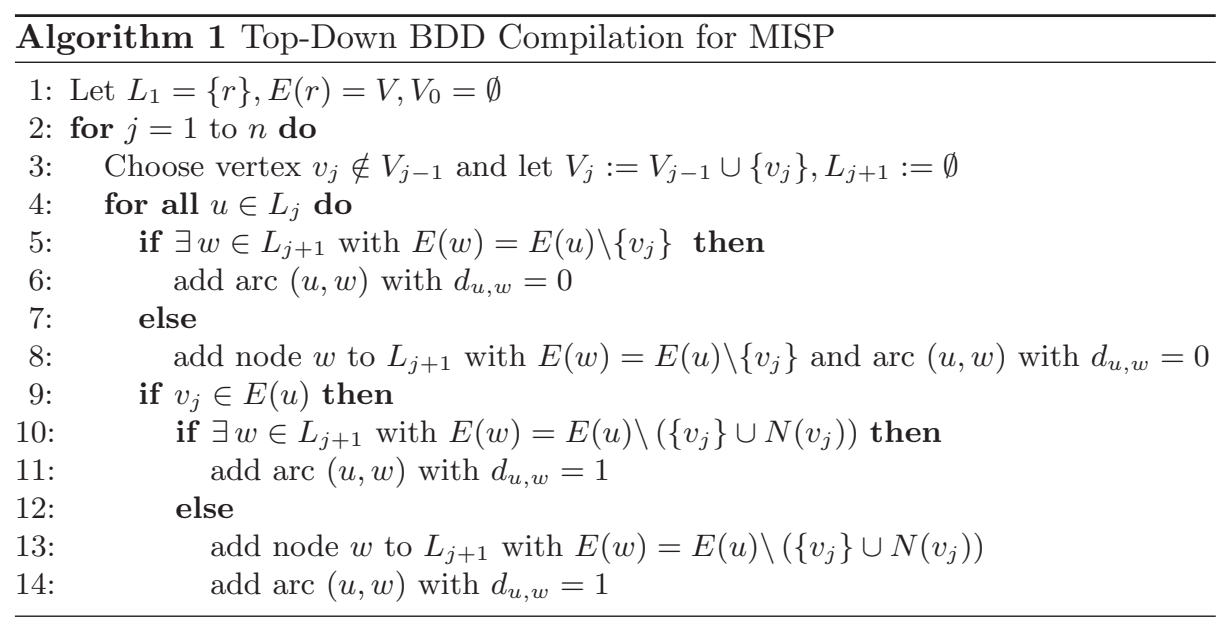

The top-down compilation for the MISP is outlined in Algorithm 1, which is a specialization of the procedure presented in [5]. We start with the root $r$ of $B$ having $E(r)=V$, since $\operatorname{Sol}\left(\left.B^{+}\right|_{L_{1}}\right)=\emptyset$ and hence all vertices in $G$ are eligible. The construction is then performed layer by layer: For each node $u \in L_{j}$, we compute the state of the nodes corresponding to the zero-arcs and the one-arcs extensions of $u$, according to $E(u)$. If there exists some node $w \in L_{j+1}$ with the same state, we add an arc $(u, w)$ to the BDD. Otherwise, we create a new node $w$ in $L_{j+1}$ and add an arc $(u, w)$. The state of the zero-arc and one-arc extensions of $u$ can be shown to be $E(u) \backslash\{u\}$ and $E(u) \backslash(\{u\} \cup N(u))$, respectively.

\section{Variable Ordering for Exact BDD Compilation}

The order of variables plays a key role in the size of exact BDDs. The impact of different orderings can be substantial, as shown in Figure 2. The example demonstrates two orderings for the graph presented in Figure 2a. The first ordering is constructed by alternating between the endpoints of the path, yielding a BDD of width 4 as depicted in Figure $2 \mathrm{~b}$. If vertices are taken according to the path order, the exact BDD has half the width, as presented in Figure 2c.

An optimal ordering minimizes the size of the exact BDD representing a given set of solutions. Previous works have focused on generating procedures to find optimal variable orderings for general BDDs (e.g., [10]). It was shown in [6] that improving a given variable ordering, in terms of reducing the size of an $\mathrm{BDD}$, is in general an NP-hard problem.

In this section we analyze variable orderings for the BDD representing the family of independent sets of a problem. We first examine particular classes of graphs, namely cliques, paths, and trees. We establish polynomial bounds on the widths (and hence size) of the exact BDDs with respect to the graph size. This is achieved by providing an ordering of the vertices that forces the width to be within a certain bound. Finally, we discuss the width for general graphs. 


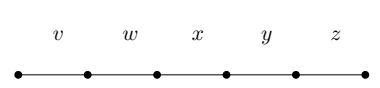

(a) Path graph

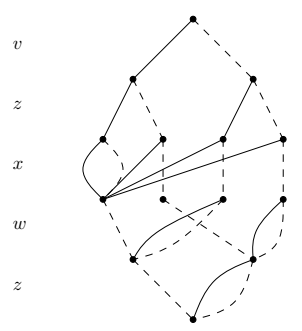

(b) Width 4

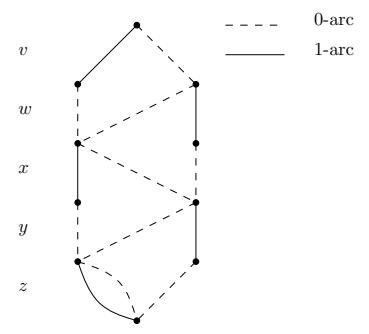

(c) Width 2

Fig. 2: Graph and exact BDD for two different orderings.

Let $S\left(L_{j}\right)$ be the set of states on nodes in $L_{j}, S\left(L_{j}\right)=\cup_{u \in L_{j}} E(u)$. To bound the width of a given layer $j$, we need only count the number of states that may arise from independent sets on $\left\{v_{1}, \ldots, v_{j-1}\right\}$. This is because each layer will have one and only one node for each possible state, and so there is a one-to-one correspondence between the number of states and the size of a layer. We now show the following Theorems.

Theorem 2. Let $G=(V, E)$ be a clique. Then, for any ordering of the vertices, the width of the exact reduced BDD will be 2 .

Proof. Consider any layer $j$. The only possible independent sets on $\left\{v_{1}, \ldots, v_{j+1}\right\}$ are $\emptyset$ or $\left\{v_{i}\right\}, i=1, \ldots, j-1$. For the former, $E\left(\emptyset \mid\left\{v_{j}, \ldots, v_{n}\right\}\right)=\left\{v_{j}, \ldots, v_{n}\right\}$ and for the latter, $E\left(\left\{v_{i}\right\} \mid\left\{v_{j}, \ldots, v_{n}\right\}\right)=\emptyset$, establishing the bound.

Theorem 3. Let $G=(V, E)$ be a path. Then, there exists an ordering of the vertices for which the width of the exact reduced BDD will be 2.

Proof. Let the ordering of the vertices be given by the positions in which they appear in the path. Consider any layer $j$. Of the remaining vertices in $G$, namely $\left\{v_{j}, \ldots, v_{n}\right\}$, the only vertex with any adjacencies to $\left\{v_{1}, \ldots, v_{j-1}\right\}$ is $v_{j}$. Therefore, for any independent set $I \subseteq\left\{v_{1}, \ldots, v_{j-1}\right\}, E\left(I \mid \bar{V}_{j-1}\right)$ will either be $\left\{v_{j}, \ldots, v_{n}\right\}$ (when $v_{j-1} \notin I$ ) and $\left\{v_{j+1}, \ldots, v_{n}\right\}$ (when $v_{j-1} \in I$ ). Therefore there can be at most 2 states in any given layer.

Theorem 4. Let $G=(V, E)$ be a tree. Then, there exists an ordering of the vertices for which the width of the exact reduced BDD will be no larger than $n$, the number of vertices in $G$.

Proof. We proceed by induction on $n$. For the base case, a tree with 2 vertices is a path, which we already know has width 2 . Now let $T$ be a tree on $n$ vertices. Any tree on $n$ vertices contains a vertex $v$ for which the connected components $C_{1}, \ldots, C_{k}$ created upon deleted $v$ from $T$ have sizes $\left|C_{i}\right| \leq \frac{n}{2}[16]$. Each of these 
connected components are trees with fewer than $\frac{n}{2}$ vertices, so by induction, there exists an ordering of the vertices on each component $C_{i}$ for which the resulting BDD $B_{i}$ will have width $\omega\left(B_{i}\right) \leq \frac{n}{2}$. For component $C_{i}$, let $v_{1}^{i}, \ldots, v_{\left|C_{i}\right|}^{i}$ be an ordering achieving this width.

Let the final ordering of the vertices in $T$ be $v_{1}^{1}, \ldots, v_{\left|C_{1}\right|}^{1}, v_{1}^{2}, \ldots, v_{\left|C_{k}\right|}^{k}, v$ which we use to create BDD $B$ for the set of independent sets in $T$. Consider layer $\ell \leq n-1$ of $B$ corresponding to vertex $v_{j}^{i}$. We claim that the only possible states in $S(\ell)$ are $s \cup C_{i+1} \cup \cdots \cup C_{k}$ and $s \cup C_{i+1} \cup \cdots \cup C_{k} \cup\{v\}$, for $s \in S^{i}(j)$, where $S^{i}(j)$ is the set of states in BDD $B_{i}$ in layer $j$. Take any independent set on the vertices $I \subseteq\left\{v_{1}^{1}, \ldots, v_{\left|C_{1}\right|}^{1}, v_{1}^{2}, \ldots, v_{j-1}^{i}\right\}$. All vertices in $I$ are independent of the vertices in $C_{i+1}, \ldots, C_{k}$, and so $E\left(I \mid\left\{v_{j}^{i}, \ldots, v_{\left|C_{i}\right|}^{i}\right\} \cup C_{i+1} \cup \cdots \cup C_{k}\right) \supseteq C_{i+1} \cup \cdots \cup C_{k}$. Now, consider $I_{i}=I \cap C_{i}$. $I_{i}$ is an independent set in the tree induced on the variables in $C_{i}$ and so it will correspond to some path in $B_{i}$ from the root of that BDD to layer $j$, ending at some node $u$. The state $s$ of node $u$ contains all of the vertices $\left\{v_{j}^{i}, \ldots, v_{\left|C_{i}\right|}^{i}\right\}$ that are independent of all vertices in $I_{i}$. As $v_{1}^{i}, \ldots, v_{j-1}^{i}$ are the only vertices in the ordering up to layer $\ell$ in $B$ that have adjacencies to any vertices in $C_{i}$, we see that the set of vertices in the state of $I$ from component $C_{i}$ are exactly $s$. Therefore, $E\left(I \mid\left\{v_{j}^{i}, \ldots, v_{\left|C_{i}\right|}^{i}\right\} \cup C_{i+1} \cup\right.$ $\left.\cdots \cup C_{k}\right) \supseteq s \cup C_{i+1} \cup \cdots \cup C_{k}$. The only remaining vertex that may be in the state is $v$, finishing the claim. Therefore, as the only possible states on layer $\ell$ are $s \cup C_{i+1} \cup \cdots \cup C_{k}$ and $s \cup C_{i+1} \cup \cdots \cup C_{k} \cup\{v\}$, for $s \in S^{i}(j)$, we see that $\omega_{\ell} \leq \frac{n}{2} \cdot 2=n$, as desired. The only layers remaining to bound is $L_{n}$ : the only possible states on layer $n$ are $\{v\}$ and $\emptyset$.

Theorem 5. Let $G=(V, E)$ be any graph. There exists an ordering of the vertices for which $\omega_{j} \leq F_{j+1}$, where $F_{k}$ is the $\mathrm{k}^{\text {th }}$ Fibonacci number.

Theorem 5 provides a bound on the width of the exact BDD for any graph. The importance of this theorem goes further than the actual bound provided on the width of the exact BDD for any graph. First, it illuminates another connection between the Fibonacci numbers and the family of independent sets of a graph, as investigated throughout the Graph Theory literature (see for example $[8,11,9,18])$. In addition to this theoretical consideration, the underlying principles in the proof provide insight into what heuristic ordering for the vertices in a graph could lead to BDDs with small width. We show in Section 6 that finding vertex orderings for which the exact BDD has small width correlates with the bound provided by relaxation BDDs using the same ordering. The ordering inspired by the underlying principle in the proof yields strong relaxation BDDs.

\section{Proof (proof of Theorem 5).}

Let $P=P^{1}, \ldots, P^{k}, P^{i}=\left\{v_{1}^{1}, \ldots, v_{i_{k}}^{1}\right\}$, be a maximal path decomposition of the vertices of $G$, where by a maximal path decomposition we mean a set of paths that partition $V$ satisfying that $v_{1}^{i}$ and $v_{i_{k}}^{i}$ are not adjacent to any vertices in $\cup_{j=i+1}^{k} P^{j}$. Hence, $P^{i}$ is a maximal path (in that no vertices can be appended to the path) in the graph induced by the vertices not in the paths, $P^{1}, \ldots, P^{i-1}$. 
Let the ordering of the vertices be given by $v_{1}^{1}, \ldots, v_{i_{1}}^{1}, v_{1}^{2}, \ldots, v_{i_{k}}^{k}$, i.e., ordered by the paths and by the order that they appear on the paths. Let the vertices also be labeled, in this order, by $y_{1}, \ldots, y_{n}$.

We proceed by induction, showing that if layers $L_{j}$ and $L_{j+1}$ have widths $\omega_{j}$ and $\omega_{j+1}$, respectively, then the width of layer $L_{j+3}$ is bounded by $\omega_{j}+2 \cdot \omega_{j+1}$, thereby proving that each layer $L_{j}$ is bounded by $F_{j+1}$ for every layer $j=$ $1, \ldots, n+1$, since $F_{j+3}=F_{j}+2 \cdot F_{j+1}$.

First we show that $L_{4}$ has width bounded by $F_{5}=5$. We can assume that $G$ is connected and has at least 4 vertices, so that $P_{1}$ has at least 3 vertices. $\omega_{1}=1$. Also, $\omega_{2}=2$, with layer $L_{2}$ having nodes $u_{1}^{2}, u_{2}^{2}$ arising from the partial solutions $I=\emptyset$ and $I=\left\{w_{1}\right\}$, respectively. The corresponding states will be $E\left(u_{1}^{2}\right)=V \backslash\left\{y_{1}\right\}$ and $E\left(u_{2}^{2}\right)=V \backslash\left(\left\{y_{1}\right\} \cup N\left(y_{1}\right)\right)$. Now, consider layer $L_{3}$. The partial solution ending at node $E\left(u_{2}^{2}\right)$ cannot have $y_{2}$ added to the independent set because $y_{2}$ does not appear in $E\left(u_{2}^{2}\right)$ since $y_{2} \in N\left(w_{1}\right)$. Therefore, there will be exact 3 outgoing arcs from the nodes in $L_{2}$. If no nodes are combined on the third layer, there will be 3 nodes $u_{i}^{3}, i=1,2,3$ with states $E\left(u_{1}^{3}\right)=V \backslash\left\{y_{1}, y_{2}\right\}$, $E\left(u_{2}^{3}\right)=V \backslash\left(\left\{y_{1}, y_{2}\right\} \cup N\left(y_{2}\right)\right)$, and $E\left(u_{3}^{3}\right)=V \backslash\left(\left\{y_{1}, y_{2}\right\} \cup N\left(y_{1}\right)\right)$. Finally, as $P^{1}$ has length at least 3 , vertex $y_{3}$ is adjacent to $y_{2}$. Therefore, we cannot add $y_{3}$ under node $u_{2}^{3}$, so layer 4 will have width at most 5 , finishing the base case.

Now let the layers of the partially constructed BDD be given by $L_{1}, \ldots, L_{j}, L_{j+1}$ with corresponding widths $\omega_{i}, i=1, \ldots, j+1$. We break down into cases based on where $y_{j+1}$ appears in the path that it belongs to in $P$, as follows.

Case 1: $y_{j+1}$ is the last vertex in the path that it belongs to. Take any node $u \in L_{j+1}$ and its associated state $E(u)$. Including or not including $y_{j+1}$ results in state $E(u) \backslash\left\{y_{j+1}\right\}$ since $y_{j+1}$ is independent of all vertices $y_{i}, i \geq j+2$. Therefore, $\omega_{j+2} \leq \omega_{j+1}$ since each arc directed out of $u$ will be directed at the same node, even if the zero-arc and the one-arc are present. And, since in any BDD $\omega_{k} \leq 2 \cdot \omega_{k-1}$, we have $\omega_{j+3} \leq 2 \cdot \omega_{j+2} \leq 2 \cdot \omega_{j+1}<\omega_{j}+2 \cdot \omega_{j+1}$.

Case 2: $y_{j+1}$ is the first vertex in the path that it belongs to. In this case, $y_{j}$ must be the last vertex in the path that it belongs to. By the reasoning in Case 1 , it follows that $\omega_{j+1} \leq \omega_{j}$. In addition, we can assume that $y_{j+1}$ is not the last vertex in the path that it belongs to because then we are in case 1. Therefore, $y_{j+2}$ is in the same path as $y_{j+1}$ in $P$. Consider $L_{j+2}$. In the worst case, each node in $L_{j+1}$ has $y_{j+1}$ in its state so that $\omega_{j+2}=2 \cdot \omega_{j+1}$. But, any node arising from a one-arc will not have $y_{j+2}$ in its state. Therefore, there are at most $\omega_{j+1}$ nodes in $L_{j+2}$ with $y_{j+2}$ in their states and at most $\omega_{j+1}$ nodes in $L_{j+2}$ without $y_{j+2}$ in their states. For the set of nodes without $y_{j+2}$ in their states, we cannot make a one-arc, showing that $\omega_{j+3} \leq \omega_{j+2}+\omega_{j+1}$. Therefore, we have $\omega_{j+3} \leq \omega_{j+1}+\omega_{j+2} \leq 3 \cdot \omega_{j+1} \leq \omega_{j}+2 \cdot \omega_{j+1}$.

Case 3: $y_{j+1}$ is not first or last in the path that it belongs to. As in case $2, \omega_{j+1} \leq 2 \cdot \omega_{j}$, with at most $\omega_{j}$ nodes on layer $L_{j+1}$ with $w_{j+2}$ in it's corresponding state label. Therefore, $L_{j+2}$ will have at most $\omega_{j}$ more nodes in it than layer $L_{j+1}$. As the same thing holds for layer $L_{j+3}$, in that it will have 
at most $\omega_{j+1}$ more nodes in it than layer $L_{j+2}$, we have $\omega_{j+3} \leq \omega_{j+2}+\omega_{j+1} \leq$ $\omega_{j+1}+\omega_{j}+\omega_{j+1}=\omega_{j}+2 \cdot \omega_{j+1}$, as desired, and finishing the proof.

We note here that using instance C2000.9 from the benchmark set discussed in Section 6, a maximal path decomposition ordering of the vertices yields widths approximately equal to the Fibonacci numbers, as seen in Table 1.

Table 1: Widths of Exact BDD for C.2000.9

\begin{tabular}{c|cccccccccccccccc}
\hline$j$ & 1 & 2 & 3 & 4 & 5 & 6 & 7 & 8 & 9 & 10 & 11 & 12 & 13 & 14 & 15 & $\cdots$ \\
\hline$w_{j}$ & 1 & 2 & 3 & 5 & 8 & 13 & 21 & 31 & 52 & 65 & 117 & 182 & 299 & 481 & 624 & $\cdots$ \\
$F i b(j+1)$ & 1 & 2 & 3 & 5 & 8 & 13 & 21 & 34 & 55 & 89 & 144 & 233 & 377 & 610 & 987 & $\cdots$ \\
\hline
\end{tabular}

\section{$5 \quad$ Variable Ordering for Relaxation BDDs}

In this section we provide heuristic orderings for the vertices to be used during the top-down compilation of relaxation BDDs. These orderings are based on the Theorems proved in Section 4, with the idea that by examining simple structured problems, we can gain intuition as to what is controlling the width of the exact BDD for general graphs, hopefully yielding tighter upper bounds. First, we describe an alteration to the top-down exact BDD compilation for the purpose of generating relaxation BDDs, and then present the heuristic orderings.

A method for generating relaxation BDDs is developed in [5]. It alters the top-down exact compilation algorithm by restricting the maximum width of the BDD according to an input parameter $W$. This is done as follows. After a layer $L_{j}$ is built, the method verifies if its width exceeds $W$. If so, nodes are merged based on some heuristic choice. Along with merging the nodes, a merging operation defined on the states of the nodes that are to be merged must be defined so that the feasible solutions are preserved during the construction of the remaining layers. For the MISP, the Theorem below defines a proper merging operation.

Theorem 6. Setting the state of the merged nodes as the union of their original states ensures that a relaxation BDD is created.

We now present the following heuristic orderings.

Maximal Path Decomposition (MPD). As show in Theorem 5, such an ordering yields an exact BDD with width bounded by the Fibonnaci numbers, yielding a theoretical worst-case bound on the width for any instance. This ordering can be pre-computed in worst-case time complexity $\mathcal{O}(|V|+|E|)$. We note that different maximal path decompositions may yield different sized BDDs.

Minimum Number of States (MIN). In this ordering, we select the next vertex in the BDD as the vertex which appears in the fewest states of the layer we are currently building. The driving force behind the proof of Theorem 5 is that 
when constructing a layer, if a vertex does not belong to the state of a node on a previous layer, we cannot include this vertex: i.e. we cannot add a one-arc, only the zero-arc. This suggests that selecting a variable appearing the fewest number of times in the states on a layer will yield a small width BDD. The worst-case time complexity to perform this selection is $\mathcal{O}(W|V|)$ per layer.

$k$-Look Ahead Ordering ( $k L A)$. This ordering can be employed for any COP. In $1 L A$, after selecting the first $j$ vertices and constructing the top $j+1$ layers, the next chosen vertex is the one that yields the smallest width for layer $j+2$ if it were selected next. This procedure can be generalize for arbitrary $k<n$ by considering subsets of yet to be selected vertices. The worst case running time for selecting a vertex can be shown to be $\mathcal{O}\left(\left(\begin{array}{l}n \\ k\end{array}\right) \cdot W|V|^{2} \log |W|\right)$ per layer.

\section{$6 \quad$ Experimental Results}

Our experiments focus on the complement graphs of the well-known DIMACS problem set for the Maximum Clique Problem, which can obtained by accessing http://dimacs.rutgers.edu/Challenges/. The experiments ran on an Intel Xeon E5345 with 8 GB RAM. The BDD was implemented in $\mathrm{C}++$.

\subsection{Exact BDDs for Trees}

The purpose of the first set of experiments is to demonstrate empirically that variable orderings potentially play a key role in the width of exact BDDs representing combinatorial optimization problems. To this end, we have selected a particular graph structure, namely trees, for which we can define an ordering yielding a polynomial bound on its width (Theorem 4). We then compare the ordering that provides this bound with a set of randomly generated orderings. We also compare with the MPD heuristic, which has a known bound for general graphs according to Theorem 5 . The trees were generated from the benchmark problems C125.9, keller4, c-fat100-1, p_hat300-1, brock200_1, and san200_0.7_1 by selecting 5 random trees each on 50 vertices from these graphs. The treespecific ordering discussed in Theorem 4 is referred to as the $C V$ (due to the computation of cut-vertices in the corresponding proof). We generated exact BDDs using 100 uniform-random orderings for each instance, and report the minimum, average, and maximum obtained widths.

The results are shown in Table 2. In all cases, none of the 100 random orderings yielded exact BDDs with width smaller than the ones generated from the CV or MPD orderings. Moreover, the average was consistently more than an order of magnitude worse than either of the structured orderings. This confirms that investigating variable orderings can have a substantial effect on the width of the exact BDDs produced for independent set problems. In addition, we see that also across all instances, the CV ordering, that is specific to trees, outperforms the MPD ordering that can be applied to general graphs, suggesting that investigating orderings specific to particular classes of instances can also have a positive impact on the width of exact BDDs. 
Table 2: Random Trees

\begin{tabular}{|c|c|c|c|c|c|c|c|c|c|c|c|}
\hline Instance & Min & Avg & Max & CV & MPD & Instance & Min & Avg & Max & CV & MPD \\
\hline p0_1.t-1 & 2336 & 22105.1 & 116736 & 16 & 160 & C125.9.t-1 & 768 & 7530.72 & 24576 & 12 & 228 \\
\hline & 672 & 8532.92 & 86016 & 16 & 312 & & 1600 & 19070 & 131072 & 12 & 528 \\
\hline & 672 & 7977.92 & 28608 & 8 & 120 & & 1024 & 8348.04 & 30720 & 12 & 288 \\
\hline & 2880 & 17292.9 & 67200 & 16 & 132 & & 736 & 4279.62 & 16704 & 16 & 312 \\
\hline & 1200 & 12795.2 & 55680 & 8 & 54 & C125.9.t-5 & 480 & 18449.3 & 221184 & 16 & 120 \\
\hline & 896 & 1776 & 221184 & 8 & 112 & & 952 & & 1152 & 8 & 248 \\
\hline & 1152 & 109 & 55040 & 16 & 144 & & 768 & .12 & 71680 & 12 & 444 \\
\hline & 2048 & 2372 & 150528 & 10 & 72 & & 2688 & 16942.1 & 74240 & 10 & 40 \\
\hline & 624 & 5883 & 46656 & 12 & 180 & & 2048 & 14297.8 & 77440 & 16 & 368 \\
\hline c-fat200-1.t-5 & 864 & 7509.66 & 27648 & 10 & 480 & keller4.t-5 & 720 & 11401.8 & 73728 & 8 & 288 \\
\hline & 792 & 1514 & 54720 & 10 & 200 & san200_0.7 & 1920 & 22771.2 & 139776 & 10 & 28 \\
\hline t-2 & 1280 & 14618.5 & 86016 & 16 & 192 & 2 & 1024 & 7841.42 & 44160 & 12 & 92 \\
\hline$. t-3$ & 624 & 11126.6 & 69120 & 12 & 138 & 3 & 768 & 8767.76 & 36864 & 8 & 88 \\
\hline & 1152 & 13822.9 & & 16 & 74 & 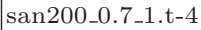 & 960 & 9981.28 & 43008 & 16 & 84 \\
\hline p_hat300-1.t-5 & 1536 & 16152 & 82944 & 14 & 160 & san200_0.7_1.t-5 & 1536 & 9301.92 & 43008 & 12 & 288 \\
\hline
\end{tabular}

\subsection{Exact BDD Width versus Relaxation BDD Bound}

The second set of experiments aims at providing an empirical evidence to the main hypothesis considered in this paper. Namely, that a problem instance with a smaller exact BDD results in a relaxation BDD that yields a tighter bound. The instances in this test were generated as follows. We first selected 5 instances from the DIMACS benchmark: brock200_1, gen200_p.0.9_55, keller4, p.hat300-2, and san200_0.7_1. Then, we uniformly at random extracted 5 connected induced subgraphs with 50 vertices for each instance, which is approximately the largest graph size that the exact BDD can be built within our memory limits.

The tests are described next. For each instance and all orderings MPD, MIN, random, and 1LA, we collected the width of the exact BDD and the bound obtained by a relaxation BDD with a maximum width of 10 (the average over 100 orderings for the random procedure). This corresponds to sampling different exact BDD widths and analyzing their respective bounds, since distinct variables orderings may yield BDDs with very different exact widths.

Figure 3 presents a scatter plot of the derived upper bound as a function of the exact widths in log-scale, also separated by the problem class from which the instance was generated. Analyzing each class separately, we observe that the bounds and width increase proportionally, reinforcing our hypothesis. In particular, this proportion tends to be somewhat constant, that is, the points tend to a linear curve for each class. We notice that this shape has different slopes according to the problem class, hence indicating that the effect of the width might be more significant for certain instances.

In Figure 4 we plot the bound as a function of the exact width for a single random instance extracted from san200_0.7_1. In this particular case, we applied a procedure that generated 1000 exact BDDs with a large range of widths: the minimum observed BDD width was 151 and the maximum was 27684, and the widths were approximately uniformly distributed in this interval. We then computed the corresponding upper-bounds for a relaxed BDD, constructed using the orderings described above, with width 10 . The width is given in a log-scale. 


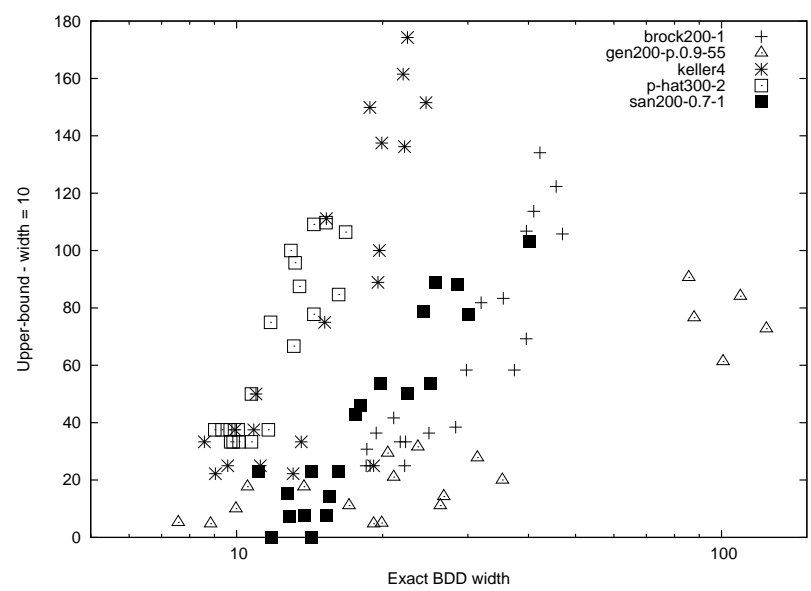

Fig. 3: Bound of relaxation BDD vs. exact BDD width.

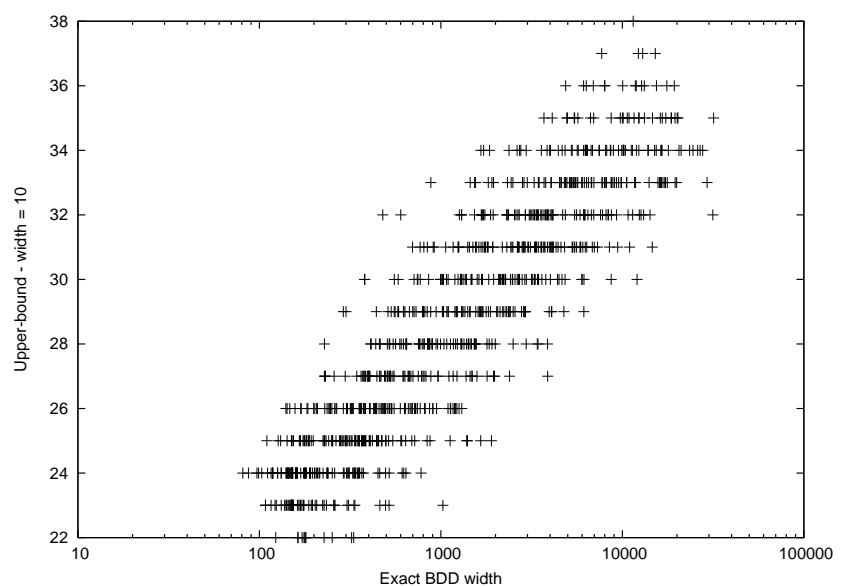

Fig. 4: Bound of relaxation BDD vs. exact BDD width for san200_0.7_1.

The Figure also shows a strong correlation between the width and the obtained bound, analogous to the previous set of experiments. A similar behavior is obtained if the same chart is plotted for other instances.

\subsection{Relaxation Bounds}

We now report the upper bound provided by the relaxation BDD for the original benchmark set, considering all heuristic orderings described in Section 5 for maximum widths 100, 500, and 1000. In addition, we generate 100 random orderings generated uniformly at random, denoted here by RAND, and the bound reported 
Table 3: Benchmark Problems Relaxations

\begin{tabular}{|c|c|c|c|c|c|c|c|c|c|c|c|c|c|c|c|}
\hline Maximum Width & & 100 & & & & 500 & & & & 1000 & & & & & 1000 \\
\hline Instance & OPT & MIN & MAX & RAND & $1 \mathrm{LA}$ & MIN & MAX & RAND & $1 \mathrm{LA}$ & MIN & MAX & RAND & $1 \mathrm{LA}$ & CPLEX (1 minute) & MIN \\
\hline C1000.9.clq & \begin{tabular}{|c|}
68 \\
2
\end{tabular} & 261 & 419 & 585.42 & 259 & 244 & 394 & 528.25 & 241 & 240 & 384 & 506.63 & 238 & 221.78 & 240 \\
\hline C125.9.clq & 34 & 46 & 55 & 71.68 & 44 & 45 & 52 & 64.51 & 42 & 43 & 50 & 61.78 & 41 & 41.2846 & 43 \\
\hline C2000.5.clq & 16 & 153 & 353 & 368.34 & 152 & 121 & 249 & 252.27 & 120 & 110 & 218 & 218 & 110 & $1.00 \mathrm{E}+75$ & 110 \\
\hline C2000.9.clq & 77 & 480 & 829 & 1170.91 & 479 & 447 & 788 & 1055.26 & 447 & 436 & 767 & 1012.4 & 433 & $1.00 \mathrm{E}+75$ & 436 \\
\hline C250.9.clq & 44 & 80 & 107 & 144.84 & 78 & 74 & 99 & 130.46 & 73 & 72 & 98 & 125.21 & 72 & 70.9322 & 72 \\
\hline C4000.5.clq & 18 & 281 & 708 & 736.31 & 280 & 223 & 497 & 504.46 & 223 & 202 & 429 & 435.31 & 203 & $1.00 \mathrm{E}+75$ & 202 \\
\hline C500.9.clq & 57 & 142 & 215 & 291.48 & 142 & 134 & 203 & 262.57 & 133 & 132 & 198 & 251.8 & 131 & 123.956 & 132 \\
\hline gen200_p0.9_44.clq & 44 & 62 & 84 & 115.69 & 62 & 61 & 79 & 103.98 & 59 & 59 & 78 & 99.78 & 56 & 44 & 59 \\
\hline gen200_p0.9_55.clq & 55 & 67 & 88 & 116.39 & 65 & 63 & 84 & 104.88 & 62 & 61 & 81 & 100.57 & 59 & 55 & 61 \\
\hline gen400_p0.9_55.clq & 55 & 100 & 168 & 233.15 & 100 & 99 & 161 & 210.21 & 96 & 94 & 156 & 201.84 & 94 & 55 & 94 \\
\hline gen400_p0.9_65.clq & 65 & 112 & 168 & 233.63 & 110 & 105 & 161 & 210.55 & 105 & 103 & 159 & 202.11 & 101 & 65 & 103 \\
\hline gen $400_{-}$p0.9_75.clq & 75 & 118 & 170 & 234.23 & 118 & 109 & 164 & 211.2 & 109 & 108 & 158 & 202.73 & 105 & 75 & 108 \\
\hline brock200_1.clq & 21 & 42 & 64 & 72.12 & 41 & 36 & 54 & 58.61 & 36 & 34 & 50 & 54.01 & 35 & 38.9817 & 34 \\
\hline brock200_2.clq & 12 & 22 & 35 & 35.6 & 22 & 17 & 24 & 24.68 & 18 & 16 & 22 & 21.69 & 16 & 22.3764 & 16 \\
\hline brock200_3.clq & 15 & 28 & 48 & 48.87 & 29 & 24 & 36 & 36.22 & 25 & 23 & 33 & 32.39 & 23 & 28.3765 & 23 \\
\hline brock200_4.clq & 17 & 32 & 53 & 56.61 & 32 & 29 & 42 & 43.32 & 27 & 26 & 37 & 39.12 & 25 & 31.5437 & 26 \\
\hline brock400_1.clq & 27 & 72 & 127 & 145.81 & 71 & 63 & 108 & 118.75 & 63 & 60 & 102 & 109.32 & 61 & 67.2201 & 60 \\
\hline brock400_2.clq & 29 & 75 & 128 & 147.35 & 72 & 63 & 107 & 119.47 & 61 & 61 & 101 & 110.16 & 60 & 67.9351 & 61 \\
\hline brock400_3.clq & 31 & 72 & 127 & 146.19 & 73 & 64 & 109 & 118.63 & 64 & 60 & 102 & 109.12 & 60 & 67.4939 & 60 \\
\hline brock400_4.clq & 33 & 70 & 129 & 146.43 & 71 & 63 & 110 & 119.54 & 63 & 63 & 106 & 109.59 & 61 & 67.3132 & 63 \\
\hline brock800_1.clq & 23 & 99 & 204 & 222.01 & 100 & 85 & 160 & 168.39 & 86 & 79 & 145 & 151.21 & 78 & 136.103 & 79 \\
\hline brock800_2.clq & 24 & 101 & 201 & 224.38 & 100 & 86 & 162 & 170.65 & 85 & 79 & 145 & 153.29 & 79 & 136.538 & 79 \\
\hline brock800_3.clq & 25 & 101 & 203 & 222.61 & 100 & 84 & 164 & 169.05 & 84 & 81 & 149 & 151.31 & 79 & 130.832 & 81 \\
\hline brock800_4.clq & 26 & 101 & 205 & 223.41 & 100 & 84 & 161 & 169.81 & 84 & 80 & 145 & 152.66 & 78 & 132.696 & 80 \\
\hline c-fat 200-1.clq & 12 & 12 & 12 & 12 & 12 & 12 & 12 & 12 & 12 & 12 & 12 & 12 & 12 & 12 & 12 \\
\hline c-fat 200-2.clq & 24 & 24 & 24 & 24 & 24 & 24 & 24 & 24 & 24 & 24 & 24 & 24 & 24 & 24 & 24 \\
\hline c-fat 200-5.clq & 58 & 58 & 58 & 58 & 58 & 58 & 58 & 58 & 58 & 58 & 58 & 58 & 58 & 61.6953 & 58 \\
\hline c-fat500-1.clq & 14 & 14 & 15 & 16.62 & 14 & 14 & 14 & 14 & 14 & 14 & 14 & 14 & 14 & 230.513 & 14 \\
\hline c-fat500-10.clq & 126 & 126 & 126 & 126 & 126 & 126 & 126 & 126 & 126 & 126 & 126 & 126 & 126 & 246 & 126 \\
\hline c-fat $500-2$.clq & 26 & 26 & 26 & 26 & 26 & 26 & 26 & 26 & 26 & 26 & 26 & 26 & 26 & 240 & 26 \\
\hline c-fat500-5.clq & 64 & 64 & 64 & 64 & 64 & 64 & 64 & 64 & 64 & 64 & 64 & 64 & 64 & 244.5 & 64 \\
\hline hamming $10-2 . c l q$ & 512 & 512 & 512 & 892.69 & 515 & 512 & 512 & 871.68 & 512 & 512 & 512 & 862.99 & 512 & 512 & 512 \\
\hline hamming10-4.clq & 40 & 106 & 91 & 456.63 & 105 & 96 & 76 & 385.13 & 93 & 79 & 72 & 359.76 & 79 & 206.047 & 79 \\
\hline hamming6-2.clq & 32 & 32 & 32 & 37.01 & 32 & 32 & 32 & 34.03 & 32 & 32 & 32 & 33.28 & 32 & 32 & 32 \\
\hline hamming6-4.clq & 4 & 4 & 4 & 5.98 & 4 & 4 & 4 & 4 & 4 & 4 & 4 & 4 & 4 & 5.33333 & 4 \\
\hline hamming8-2.clq & 128 & 128 & 128 & 194.42 & 128 & 128 & 128 & 184.51 & 128 & 128 & 128 & 180.71 & 128 & 128 & 128 \\
\hline hamming8-4.clq & 16 & 20 & 21 & 62.23 & 19 & 18 & 18 & 45.66 & 18 & 17 & 17 & 40.56 & 17 & 16 & 17 \\
\hline johnson $16-2-4 . c l q$ & 8 & 11 & 11 & 38.75 & 11 & 9 & 9 & 29.24 & 9 & 8 & 8 & 25.64 & 8 & 8 & 8 \\
\hline johnson $32-2-4 . c l q$ & 16 & 40 & 35 & 250.07 & 42 & 38 & 29 & 215.06 & 39 & 35 & 25 & 202.36 & 40 & 16 & 35 \\
\hline johnson8-2-4.clq & 4 & 4 & 4 & 4 & 4 & 4 & 4 & 4 & 4 & 4 & 4 & 4 & 4 & 4 & 4 \\
\hline johnson8-4-4.clq & 14 & 14 & 15 & 24.57 & 14 & 14 & 14 & 19.82 & 14 & 14 & 14 & 18.54 & 14 & 14 & 14 \\
\hline & 11 & 19 & 22 & 43.38 & 18 & 16 & 17 & 31.24 & 16 & 15 & 16 & 27.54 & 15 & 14.75 & 15 \\
\hline & 27 & 58 & 98 & 280.74 & 59 & 56 & 77 & 225.75 & 55 & 48 & 72 & 207.08 & 49 & 32.875 & 48 \\
\hline keller6.clq & 59 & 171 & 417 & 1503.26 & 174 & 142 & 332 & 1277.98 & 144 & 123 & 307 & 1197.76 & 125 & $1.00 \mathrm{E}+75$ & 123 \\
\hline MANN_a27.clq & 126 & 142 & 138 & 327.2 & 135 & 140 & 137 & 318.93 & 137 & 139 & 137 & 315.25 & 136 & 133.331 & 139 \\
\hline MANN_a45.clq & 345 & 371 & 365 & 954.51 & 366 & 368 & 362 & 942.45 & 363 & 368 & 362 & 937.06 & 365 & 357.162 & 368 \\
\hline MANN_a81.clq & 1100 & 1154 & 1143 & 3186.21 & 1141 & 1150 & 1143 & 3166.06 & 1143 & 1148 & 1143 & 3158.78 & 1141 & 1131.82 & 1148 \\
\hline MANN_a9.clq & 16 & 18 & 18 & 27.21 & 17 & 16 & 16 & 23.9 & 16 & 16 & 16 & & 16 & 17 & 16 \\
\hline p_hat 1000-1.clq & 10 & 47 & 86 & 88.73 & 48 & 35 & 52 & 52.71 & 36 & 31 & 43 & 43.37 & 31 & 413.5 & 31 \\
\hline p_hat $1000-2 . c l q$ & 46 & 130 & 210 & 225.57 & 129 & 116 & 171 & 178.1 & 112 & 112 & 159 & 163.47 & 108 & 376.5 & 112 \\
\hline t $1000-3 . c l q$ & 68 & 202 & 324 & 383.76 & 197 & 187 & 286 & 322.62 & 179 & 179 & 272 & 302.07 & 175 & 245.674 & 179 \\
\hline p_hat 1500-1.clq & 12 & 68 & 136 & 139.02 & 68 & 51 & 83 & 83.08 & 51 & 46 & 69 & 68.33 & 45 & $1.00 \mathrm{E}+75$ & 46 \\
\hline p_hat $1500-2 . c l q$ & 65 & 199 & 344 & 357.01 & 193 & 176 & 285 & 286.03 & 174 & 168 & 267 & 263.95 & 163 & $1.00 \mathrm{E}+75$ & 168 \\
\hline & 94 & 298 & 511 & 594.04 & 296 & 277 & 452 & 502.22 & 270 & 272 & 433 & 470.91 & 266 & $1.00 \mathrm{E}+75$ & 272 \\
\hline t $300-1 . c l q$ & 8 & 17 & 27 & 26.05 & 18 & 14 & 16 & 15.89 & 14 & 12 & 13 & 13.39 & 12 & 18.2278 & 12 \\
\hline at $300-2 . \mathrm{clq}$ & 25 & 48 & 64 & 66.46 & 45 & 42 & 51 & 52.29 & 40 & 40 & 48 & 47.83 & 39 & 35.2878 & 40 \\
\hline at $300-3 . c l q$ & 36 & 70 & 99 & 114.66 & 67 & 65 & 89 & 95.93 & 61 & 62 & 84 & 89.86 & 60 & 55.2598 & 62 \\
\hline p_l & 9 & 28 & 45 & 45.33 & 27 & 21 & 28 & 27.3 & 21 & 1 & 23 & 22.7 & 19 & 158 & 18 \\
\hline 500-2.clq & 36 & 77 & 112 & 116.55 & 72 & 69 & 92 & 92.8 & 64 & 66 & 84 & 85.54 & 63 & 160.25 & 66 \\
\hline t500-3.clq & 50 & 111 & 172 & 195.67 & 109 & 106 & 155 & 165.35 & 102 & 104 & 147 & 154.88 & 99 & 90.7331 & 104 \\
\hline $700-1 . \mathrm{clq}$ & 11 & 36 & 62 & 63.27 & 36 & 27 & 39 & 37.83 & 27 & 24 & 31 & 31.33 & 24 & 272 & 24 \\
\hline t $700-2 . c l q$ & 44 & 101 & 155 & 163.0 & 99 & 90 & 128 & 130. & 88 & 8 & 118 & 120.19 & 83 & 272 & 85 \\
\hline at $700-3 . c l q$ & 62 & 153 & 234 & 272.83 & 147 & 142 & 208 & 230.14 & 141 & 137 & 198 & 215.93 & 134 & 160.333 & 137 \\
\hline 1000.clq & 15 & 28 & 184 & 202.02 & 26 & 21 & 101 & 104.09 & 19 & 19 & 78 & 79.84 & 19 & 462.5 & 19 \\
\hline 00_0.7_1.clq & 30 & 32 & 66 & 73.67 & 31 & 30 & 57 & 60.3 & 30 & & 52 & 55.37 & 30 & 30 & 30 \\
\hline _2.clq & 18 & 23 & 58 & & 21 & 20 & 48 & 56 & 20 & 19 & 46 & 50.23 & 18 & 18 & 19 \\
\hline & 70 & 71 & 86 & & 70 & 70 & 82 & & 70 & 70 & 81 & 105.13 & 70 & 70 & 70 \\
\hline $\operatorname{san} 200 \_0.9 \_2 . c l q$ & 60 & 68 & 86 & 116.48 & 64 & 64 & 83 & 105.39 & 60 & 60 & 81 & 101.05 & 60 & 60 & 60 \\
\hline san200_0.9_3.clq & 44 & 57 & 84 & 115 & 54 & 55 & 78 & 103.23 & 53 & 51 & 77 & 99 & 52 & 44 & 51 \\
\hline$x^{-1}-1$ & 13 & 17 & 66 & 69.02 & 18 & 14 & 35 & 35.6 & 14 & 13 & 28 & 28.31 & 13 & 13 & 13 \\
\hline & 40 & 50 & 142 & 160 . & 51 & 4 & 127 & & 4 & 4 & 11 & 126 & & & 42 \\
\hline$-0.7 \_2 . c$ & 30 & 44 & 12 & & 45 & 3 & 10 & & 3 & 3 & 10 & & 3 & 3 & 37 \\
\hline $\operatorname{san} 400 \_0.7 \_3 . c l q$ & 22 & 36 & 118 & 137.72 & 38 & 29 & 98 & 108.29 & 31 & 29 & 91 & 97.98 & 29 & 22 & 29 \\
\hline $\operatorname{san} 400$-0.9_1.clq & 100 & 117 & 175 & 236.22 & 118 & 109 & 169 & 214.05 & 108 & 108 & 164 & 205.73 & 108 & 100 & 108 \\
\hline & 18 & 34 & 58 & 63 & 36 & 31 & 46 & 49.56 & 32 & 30 & 44 & 45.18 & 29 & 34.5339 & 30 \\
\hline sanr200_0.9.clq & 42 & 67 & 86 & 114.78 & 66 & 63 & 83 & 103.25 & 60 & 61 & 80 & 98.89 & 61 & 59.5252 & 61 \\
\hline & 13 & 40 & 70 & 73.32 & 39 & 33 & 50 & 50.5 & 31 & 29 & 45 & 43.73 & 29 & 43.1544 & 29 \\
\hline sanr400_0.7.clq & 21 & 64 & 115 & $\begin{array}{l}12.02 \\
128.44\end{array}$ & 64 & 55 & 96 & 101.06 & 54 & 52 & 89 & 91.69 & 52 & 62.078 & 52 \\
\hline
\end{tabular}


is obtained by taking the average over the 100 generated orderings. The average compilation time for maximum width 100,500 and 1000 were $0.21,1.49$, and 3.01 seconds, respectively, for the MIN ordering (which was similar to RAND and MPD), while the average time for maximum width 100, 500, and 1000 were $65.01,318.68$, and 659.02 , respectively, for the 1LA ordering. For comparison purposes, we have also included the upper bound obtained by considering the IP formulation of the MISP, since this corresponds to a well-known bounding technique for general domains. We ran these instances with CPLEX 12.2 with default settings and took the resulting bound obtained after the root node was computed. We impose a time limit of 60 seconds so that the results were comparable to the MIN ordering with width 1000 since the longest time to create any relaxation BDD with these parameters was C.4000.5, which took 50.42 seconds.

The results are presented in Table 3 . We report for each instance the optimal or the best known feasible solution and the bounds, where CPLEX is the bound obtained by the root node relaxation using CPLEX (the symbol $+\infty$ indicates that a bound was not obtained in the 60 seconds time-limit). By first comparing the results obtained between orderings, we see that the MIN ordering and the general purpose 1LA heuristic provide the best bounds for most instances. We highlight here that the MIN and 1LA were the heuristics that provided the smallest BDD widths for the instances tested in Section 6.2. We note that MIN is generates BDDs an average of an order of magnitude faster than 1LA.

To compare the obtained bounds with CPLEX, we consider the relative bound measure, which is given by (upper bound/optimum). The average relative bound for CPLEX (omitting the instances for which CPLEX was unable to provide a bound) is given by 3.85, while for MIN and 1LA they are given by 2.34 and 2.32 , respectively, for a width of 100; and 1.92 and 1.90, respectively, for a width of 1000 (the averages are not significantly different at the $5 \%$ level between MIN and 1LA). The average relative ordering for RAND was 5.51 and 4.25 for widths of 100 and 1000, respectively. This indicates that variable orderings are crucial to obtain tighter and relevant bounds, which showed to be particularly significant for larger instances when comparing with CPLEX, explaining the smaller average relative bound. We further observe that, since times were very small for the structured heuristics, the bounds obtained here can be improved using the general purpose bound improving procedures in [5].

\section{Conclusion}

In this paper we analyzed the impact of variable ordering on the quality of the relaxation provided by binary decision diagrams. We focus on the Maximum Independent Set Problem, providing theoretical bounds on the BDD width for general and particular classes of graphs. In addition, we utilize the developed theory to propose specific and general-purpose variable ordering heuristics. Experimental results indicate that there is a strong correlation between variable ordering heuristics that yield small-sized exact BDDs and the bounds obtained by relaxed BDDs that use these orderings. 


\section{References}

1. S. B. Akers. Binary decision diagrams. IEEE Transactions on Computers, C27:509-516, 1978.

2. H. R. Andersen, T. Hadzic, J. N. Hooker, and P. Tiedemann. A constraint store based on multivalued decision diagrams. In C. Bessière, editor, Principles and Practice of Constraint Programming (CP 2007), volume 4741 of Lecture Notes in Computer Science, pages 118-132. Springer, 2007.

3. B. Becker, M. Behle, F. Eisenbrand, and R. Wimmer. BDDs in a branch and cut framework. In S. Nikoletseas, editor, Experimental and Efficient Algorithms, Proceedings of the 4th International Workshop on Efficient and Experimental Algorithms (WEA 05), volume 3503 of Lecture Notes in Computer Science, pages 452-463. Springer, 2005.

4. Markus Behle and Friedrich Eisenbrand. 0/1 vertex and facet enumeration with bdds. In ALENEX. SIAM, 2007.

5. David Bergman, Willem Jan van Hoeve, and John N. Hooker. Manipulating mdd relaxations for combinatorial optimization. In Tobias Achterberg and J. Christopher Beck, editors, CPAIOR, volume 6697 of Lecture Notes in Computer Science, pages 20-35. Springer, 2011.

6. Bollig and Wegener. Improving the variable ordering of OBDDs is NP-complete. IEEETC: IEEE Transactions on Computers, 45, 1996.

7. R. E. Bryant. Graph-based algorithms for boolean function manipulation. IEEE Transactions on Computers, C-35:677-691, 1986.

8. Neil J. Calkin and Herbert S. Wilf. The number of independent sets in a grid graph. SIAM J. Discrete Math., 11(1):54-60, 1998.

9. Martin E. Dyer, Alan M. Frieze, and Mark Jerrum. On counting independent sets in sparse graphs. SIAM J. Comput., 31(5):1527-1541, 2002.

10. Rudiger Ebendt, Wolfgang Gunther, and Rolf Drechsler. An improved branch and bound algorithm for exact BDD minimization. IEEE Trans. on CAD of Integrated Circuits and Systems, 22(12):1657-1663, 2003.

11. Florence Forbes and Bernard Ycart. Counting stable sets on cartesian products of graphs. Discrete Mathematics, 186(1-3):105-116, 1998.

12. T. Hadzic and J. N. Hooker. Postoptimality analysis for integer programming using binary decision diagrams, presented at GICOLAG workshop (Global Optimization: Integrating Convexity, Optimization, Logic Programming, and Computational Algebraic Geometry), Vienna. Technical report, Carnegie Mellon University, 2006.

13. T. Hadzic and J. N. Hooker. Cost-bounded binary decision diagrams for 0-1 programming. Technical report, Carnegie Mellon University, 2007.

14. T. Hadzic, J. N. Hooker, B. O'Sullivan, and P. Tiedemann. Approximate compilation of constraints into multivalued decision diagrams. In P. J. Stuckey, editor, Principles and Practice of Constraint Programming (CP 2008), volume 5202 of Lecture Notes in Computer Science, pages 448-462. Springer, 2008.

15. S. Hoda, W.-J. van Hoeve, and John N. Hooker. A systematic approach to mddbased constraint programming. In Proceedings of the 16th International Conference on Principles and Practices of Constraint Programming, Lecture Notes in Computer Science. Springer, 2010.

16. C. Jordan. Sur les assemblages de lignes. J. Reine Angew Math, 70:185-190, 1869.

17. C. Y. Lee. Representation of switching circuits by binary-decision programs. Bell Systems Technical Journal, 38:985-999, 1959.

18. Yufei Zhao. The number of independent sets in a regular graph. Combinatorics, Probability \&S Computing, 19(2):315-320, 2010. 